\title{
Architecture and implementation of an agent-based simulation tool for market-based pricing in Next-Generation Wireless Networks
}

\author{
Matthias Roggendorf, Fernando Beltrán, Jairo Gutiérrez ${ }^{\dagger}$ \\ University of Auckland Business School \\ 7 Symonds Street, Auckland, New Zealand \\ Email: \{m.roggendorf, f.beltran, j.gutierrez $\}$ auckland.ac.nz \\ ${ }^{\dagger}$ Currently visiting the Universitat Politècnica de Cataluyna, Barcelona
}

\begin{abstract}
- we present a generic, agent-based simulation environment for dynamic pricing in next-generation wireless networks. While a lot of effort has been put into simulation platforms for recreating the behaviour of IP-based traffic in fixed and wireless networks, no standard platform for simulating different pricing schemes in such networks has yet emerged. Our work is driven by the vision of a ubiquitous wireless network environment, in which users can dynamically request network resources for various uses from different, potentially competing network providers. For such a scenario, new pricing approaches are needed to charge the user according to dynamic factors such as current congestion levels or the the number of customers present at a specific location. The developed simulation environment serves as a generic tool for implementing and testing different pricing approaches.
\end{abstract}

\section{INTRODUCTION}

The use of Internet Protocol (IP)-based applications for the delivery of services over digital networks is one of the driving forces in the current telecommunications landscape. The introduction of IP for packet switching, routing and transportation of digital information in data communication networks has opened up a tremendously broad range of possibilities for the creation of innovative services.

Wireless networks are no exception to this trend; traditional cellular telephony providers as well as new entrants are already operating IP-based services in networks of the second and third generation, also known as $2.5 \mathrm{G}$ and $3 \mathrm{G}$, respectively. The next generation of wireless networks promises to be an all-IP ubiquitous network, merging voice and data communications into one common network [1].

Mobile networks beyond $2.5 \mathrm{G}$ and $3 \mathrm{G}$, as well as technology emerging from the data communication frontier, such as WiMAX, are seen as enablers for the seamless integration of many different access technologies, adaptable to services and applications, with high-performance and ubiquitous physical layers. These factors contribute to achieving integration, flexibility and efficiency, the promised features of a NextGeneration Wireless Network [2].

[3] suggest that as Next-Generation Wireless Networks will provide a seamless, multiple choice access to IP-based networks for mobile and fixed users, providers' business models also have to adapt to this new situation. In fact, the widely used long-term subscription models may be complemented by more flexible pricing schemes allowing users to individually negotiate access to a network and to pay a service provision, which is only charged during active connection.

One of the challenges in such a scenario is the development of suitable pricing models to support the dynamic access to wireless networks. When customers gain access to networks in a flexible fashion, static pricing plans are regarded as too inflexible because they cannot capture the particular situation in a network. Instead, parameters such as current usage level, users in the system, competition in the specific coverage area or channel quality may be used as indicators for an appropriate pricing level. Pricing schemes consisting of a flat fee provide wrong incentives for resource utilisation [4]. Such schemes risk rendering the network inefficient as users, unaware of their impact on the efficient utilization of resources, tend to behave as the exploiters of a common resource with the known consequences of overconsumption.

Today's access models for ad-hoc network admission, as currently used for WiFi access at airports or public places, require customers to manually compare tariff schemes of different carriers and to select the offer best suiting their personal needs. Moreover, such models do not allow for the introduction of differentiated services, which are expected to be one major source of revenue in the next generation of wireless networks.

\section{Motivation For THE DEVElopment OF A NEW SIMULATION ENVIRONMENT}

The existing landscape of network simulation tools was considered as not suitable for the task of testing different negotiation techniques in a setting that also included different access points belonging to competing carriers. The intended experiments are focused on the bidding process between independently acting users instead of the generation and management of network traffic in wireless networks. In contrast to an engineering approach to resource allocation, such as the implementation of a medium access control (MAC) layer, in which the same algorithm for resource allocation is used at each device to manage the traffic load, every individual user in the system may follow different goals. Additionally, 
individuals can have very different valuations for a network resource to be allocated. One group of agents might value a small network resource share as very valuable, while others might consider it useless. To model such a behaviour, each agent needs to implement his individual valuation in order to fulfill his task of negotiating for resources. Following standard economic principles, market participants are modeled using a utility function and implement different patterns of behaviour. Agents base their decisions for future actions on signals received from the market environment. Examples of market signals observable by users are the requests submitted by other agents or the recent resource allocation communicated by the provider. We assume that all agents, given their individual restrictions and external constraints, evaluate their utility functions and behave such that they maximize the expected payoff. Individual restrictions could be, for example, budget limitations, limitations in computational power to calculate negotiation strategies, or an agent's capabilities of using different wireless technologies. The network structure, or the total network capacity available, can be seen as external constraints that cannot be directly influenced by the individual agent. By using agents in studying market dynamics, heterogeneous, boundedly-rational, and adaptive behaviour of individuals can be represented and its impact on market dynamics assessed.

To model such a negotiation process for resource allocation, the simulation needs to abstract a few aspects from the many factors underlying the network traffic. It is assumed that lower level functions such as power allocation and queueing management are performed by the underlying network layers in a fashion that is transparent to the pricing layer above. Open interfaces such as those promoted by various industry groups (OSA [5]; Parlay [6]) can be adapted to provide the pricing layer with parameters such as minimum and maximum constraints of the wireless channel for resource allocation on a higher level of abstraction. Therefore, the simulation environment can concentrate on the actual negotiation process which is driven by the self-interest of independently acting agents.

Simulation is a mature technology for focusing on specific problems of networks in a particular environment. It is especially useful in the areas of network planning where "interesting" scenarios are too difficult to be represented using any other research methodology. The value of this technology, however, is directly proportional to the level of detail introduced in the model. In other words, successful simulation requires a complex description of the network operation as well as an identification of the desired performance metrics [7]. This characteristic guarantees good results for the target environment, but narrows the effectiveness of the tool. Any accurate simulation model will require heavy customisation when used with different network environments and any tool or test-bed that facilitates that outcome is indeed good news for the networking research community.

\section{THE ARCHITECTURE OF THE SIMULATION ENVIRONMENT}

The simulation environment has been developed in JAVA JDK5.0, using the Java Agent DEvelopment (JADE) Frame- work $^{1}$. JADE is open-source middleware developed jointly by CSELT (Centro Studi e Laboratori Telecommunicazioni) in conjunction with the Computer Engineering Group of the University of Parma and is used in numerous academic and industrial applications worldwide. JADE is well documented with many programming tutorials and code examples available from different sources. An active user community of over a thousand members from academic environments, as well as from from R\&D centers of world leading companies such as Motorola, HP, Siemens and Rockwell Automation, contributes to the project by providing library extensions and add-ons.

JADE simplifies the implementation of multi-agent systems through a predefined middleware concept, which fully complies with the FIPA reference model [8]. The environment itself does not provide specific agent architectures but instead provides a basic set of functionalities, which are needed in an autonomous agent implementations. JADE defines a common agent base-class for creating user-defined agents extending the standard functionality. JADE includes both the libraries required to develop application agents and the runtime environment that provides the basic services and that must be active on the device before agents can be executed.

Agent tasks in JADE are implemented as behaviours extending standard behaviour classes. Once an agent has been created and is set active, behaviours are executed based on a round nonpreemptive scheduling policy [8]. The invocation of the implemented behaviours can be triggered in different ways. For example, a behaviour can be started when a message matching certain criteria arrives at the inbox. Another possibility is to define a predefined time period after which the behaviour is started. Behaviours can also be nested and sub-behaviours can be defined. In this way an agent can either be reactive (taking an action as soon as an external event is observed) or active (start an action based of a change of an internal state).

The communication between agents is based on the FIPAdefined Message Transport Protocol (MTP) over which Agent Communication Language (ACL) messages can be sent. The format of the message content can implement an application specific ontology based on FIPA specifications for content languages (CL). Several generic JAVA interfaces can be implemented to define an application-specific ontology for agent communication. JADE provides many different transportation encodings such as XML, RDF, or proprietary bit-efficient technologies. Additionally, messages can be secured by several encryption techniques which may be important in a distributed system spanning several networks.

\section{A. Entities of the simulation environment}

The simulation environment consists of three groups of different agent types resembling a standard three-tier architecture (Figure 1). The first group, called Boundary Agents, provides the graphical user interface for the simulation setup and monitoring. Two agent types have been implemented, the GUISetupAgent and the GUIMonitorAgent. The setup agent serves as the main configuration tool for defining

\footnotetext{
${ }^{1}$ http://jade.tilab.com
} 


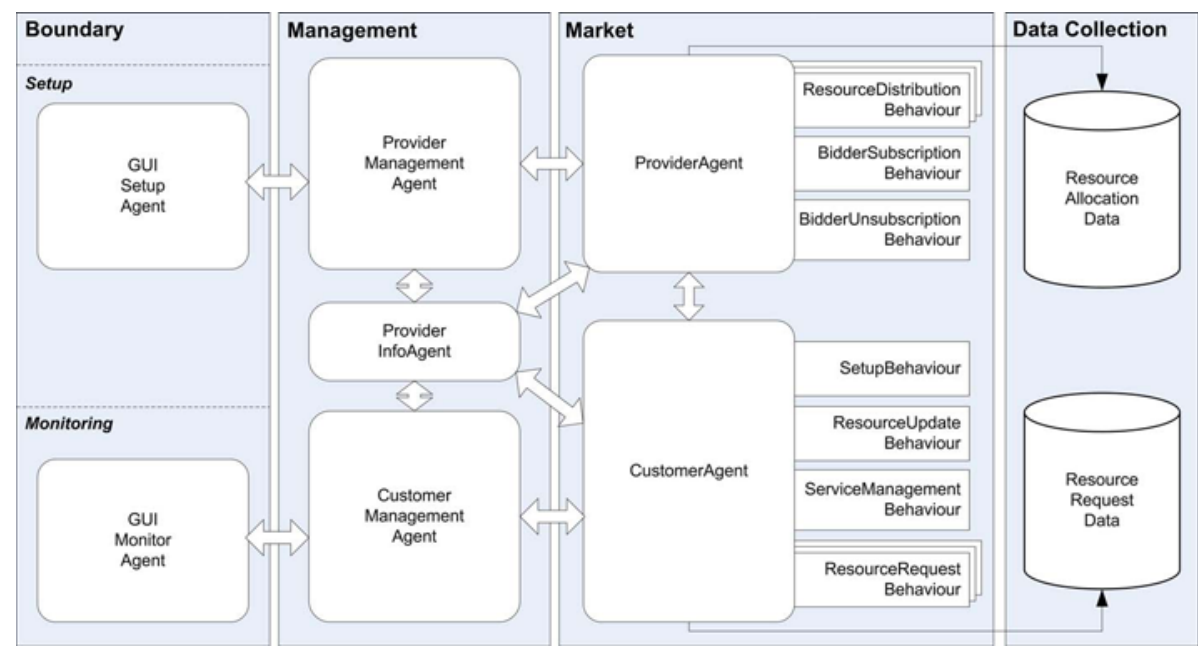

Fig. 1. Agent types of the simulation environment

the simulation scenario. A simulation scenario contains all parameters to set up the agents for an experiment. For example, for a bidder agent, the agent's valuation function needs to be speci ed as well as the agent's position and capabilities in terms of the support for different wireless technologies and billing relationships with network providers.

The second group of agents are Management Agents, which are responsible for setting up and maintaining the agent population used to run the experiments. Three types of management agents have been designed to re ect the two agent population groups and to distribute the load during the setup phase. An additional advantage of this separation is that the knowledge about agent types becomes distributed and no central entity with total knowledge about agent preferences exists. The CustomerManagementAgent is responsible for the setup of the customer population and the distribution of service requests to those agents. Since providers are assumed to be at a xed location and do not change their properties during a simulation experiment the task of the ProviderManagementAgent is to simply set up the provider population at simulation startup time and ensure the removal of the agent population when the simulation is stopped. Finally, the ProviderInfoAgent, which extends the functionality of the DFAgent, provides a service discovery function for all customer agents.

The third group of agent types, the Market Agents, represent the active agent population in the experiment. CustomerAgents are equipped with a preference description (the valuation of an agent) and goals (to ful 11 a service request as soon as they are informed by the CustomerManagementAgent). ProviderAgents hold network resources, which can be allocated to customer agents during the negotiation process. Market agents have the most modular design, to allow for an easy extension of the core functionality. Their negotiation behaviour has been left generic in the simulation environment to allow for different forms of negotiation.

\section{B. Data collection and visualisation}

Each agent in the simulation environment inherits methods for capturing simulation data at runtime. At startup, each agent reports the data structure of the data to be collected. This structure is then created in a SQL database with a unique timestamp of the simulation experiment. During the simulation each agent can write results to its table by transfering the data to a reporting behaviour. This behaviour runs as a separate thread and has access to the database.

The GUIMonitorAgent has reading rights to all data structures of the speci c simulation experiment and can visualise the data during or after runtime. It also serves as a conversion tool for exporting simulation data to other structures such as Excel les.

While this approach provides a high degree of exibility in collecting simulation data it consumes a signi cant amount of system resources especially with more complex simulation scenarios. It is therefore necessary to carefully select the reporting entities to keep the system load for reporting on a low level while at the same time capturing the important datasets.

\section{The simulation setup phase}

During the setup phase the simulation parameters for the simulation run are de ned. The GUISetupAgent implements a graphical user interface for manually de ning all the simulation setup. Alternatively, the simulation setup can be de ned in plain text les, which can be read by the agent. In the next version of the simulator, input data will be stored in XML format to allow for greater exibility in the setup parameters.

The simulation setup consists of parameters in three areas: general simulation parameters such as update intervals and threshold values, the setup of customer agents and the setup of provider agents. The latter two categories comprise parameters such as the agent's position, the transmission range, and the wireless technology used by the agent. Customer agents additionally implement a utility type, which de nes their valuation for different amounts of network resources. Table I 
TABLE I

CUSTOMISATION PARAMETERS FOR THE CustomerAgent AND THE

ProviderAgent. THE PARAMETERS MARKED WITH * ARE

MULTI-DIMENSIONAL AND MAY CONTAIN SEVERAL SUB-PARAMETERS DEPENDING ON THE SIMULATION SETUP.

\begin{tabular}{ll} 
CustomerAgent & ProviderAgent \\
\hline CustomerID & $\begin{array}{l}\text { ProviderID } \\
\text { ProviderCompany } \\
\text { XLocation } \\
\text { YLocation } \\
\text { YLocation }\end{array}$ \\
$\begin{array}{l}\text { XYRange } \\
\text { SubscriptionCompany* }\end{array}$ \\
WirelessTechnology* & $\begin{array}{l}\text { WirelessTechnology } \\
\text { ResourceDescription* }\end{array}$ \\
ActivationTime & \\
DeactivationTime & \\
UtilityType & \\
UtitityParameter* & \\
ServiceRequestType & \\
ServiceRequestParameters* & \\
BehaviourType* & \\
MovementType & \\
MovementParameter* & \\
DataCollEnabled & DataCollEnabled \\
UserDe ned* & UserDe ned* \\
\hline
\end{tabular}

provides an overview of the parameter categories for the two market agent types.

The de ned parameters are part of the simulation ontology in the form of an object-oriented data structure. An ontology is a description of concepts and relationships that can exist for a community of agents. Each object summarises several logically related parameters, which can be used as message content between agents. For example, the CustomerManagementAgent informs CustomerAgents about their type in the form of a CustomerType ontology object. The ontology concept is arbitrarily extensible if special negotiation formats require additional variables or data structures for message exchange or the initialisation of the simulation environment.

\section{The simulation phase}

After all parameters have been de ned within the graphical user interface of the SetupGUIAgent the actual simulation experiment can be started. Figure 2 shows the process of setting up all agents and the basic message exchange, which is required to initialise the simulation platform. In the rst step the CustomerManagementAgent and the ProviderManagementAgent are created and activated. In the second step all market agents are populated through the two management agents.

ProviderAgents subscribe to the ProviderInfoAgent for the publication of their parameters describing the wireless resource (e.g., transmission range, wireless technology, provider association, resource negotiation protocol). Once the CustomerAgents have been populated and have been activated to request services they can make use of the yellow page service of the ProviderInfoAgent to identify the provider agents in their range and with a compatible wireless technology. Customer agents subsequently start to send requests to provider agents to express their demand. Depending on the speci c negotiation mechanism the resource allocation is con rmed by the provider agent. A customer agent may either send requests out simultaneously to different providers, or may issue requests sequentially, by waiting for the results from one provider rst. CustomerAgents may also be able to bundle resources from different providers to increase their throughput.

\section{FIRST EXPERIMENTAL IMPLEMENTATION}

The rst stable version of the simulation environment has been used for implementing an auction-based pricing system for allocating resources in a competitive provider environment. In general, auctions are mechanisms for resource allocation in which each agent submits bids and the outcome is computed by the seller. The objective of such a mechanism is to maximise a measure of welfare [9]; provider's revenue maximisation is one example. An auctions is a powerful tool in cases where the seller of a resource is unaware of the bidders' valuation for the resources being sold. If a seller knows the valuation of a bidder he could just charge the highest possible amount to the agent. The uncertainty regarding values facing both sellers and bidders is an inherent feature of auctions [10].

Our protocol uses a second-price auction for arbitrarily divisible resources. The type of auction, called the Progressive Second-Price (PSP) auction, was introduced by Semret in 1999 for use in xed networks with one seller [11]. It inherits the properties of a second-price auction for the single-object case. The most important properties are economic efficiency and incentive compatibility. Ef ciency ensures that resources are allocated to agents with the highest valuation. Incentive compatibility motivates agents to reveal their true value for a resource instead of shading their bid or using complicated bidding strategies to gain advantage against other bidders.

Our implementation extends the concept of PSP to the situation of a wireless network setting in which customers have the choice between different network providers. With the PSP auction mechanism, customer agents express their demand with a bid $s$ represented by the a resource share $q$ and a unit price $p$. Provider agents independently run PSP auctions to allocate resources based on the bids received from customer agents. After each auction the new allocation is propagated to all customer agents.

The main challenge in the setting of multiple providers is the de nition of a bidding strategy that retains the properties of the one-provider case. The goal of each bidder agent is to maximise its utility from the resources gained from different auction markets. However, it needs to coordinate the bids on several auctions, which need to conform with the agent's valuation for the resource.

We have developed a bidding strategy, called BalancedBid, which enables a bidder to rst determine the optimal demand when faced with multiple auctions. Subsequently, the bidder calculates balanced sub-bids, which can be submitted to each single auction market. Using such a bidding strategy leads to a Nash-Equilibrium after a convergence phase. In 


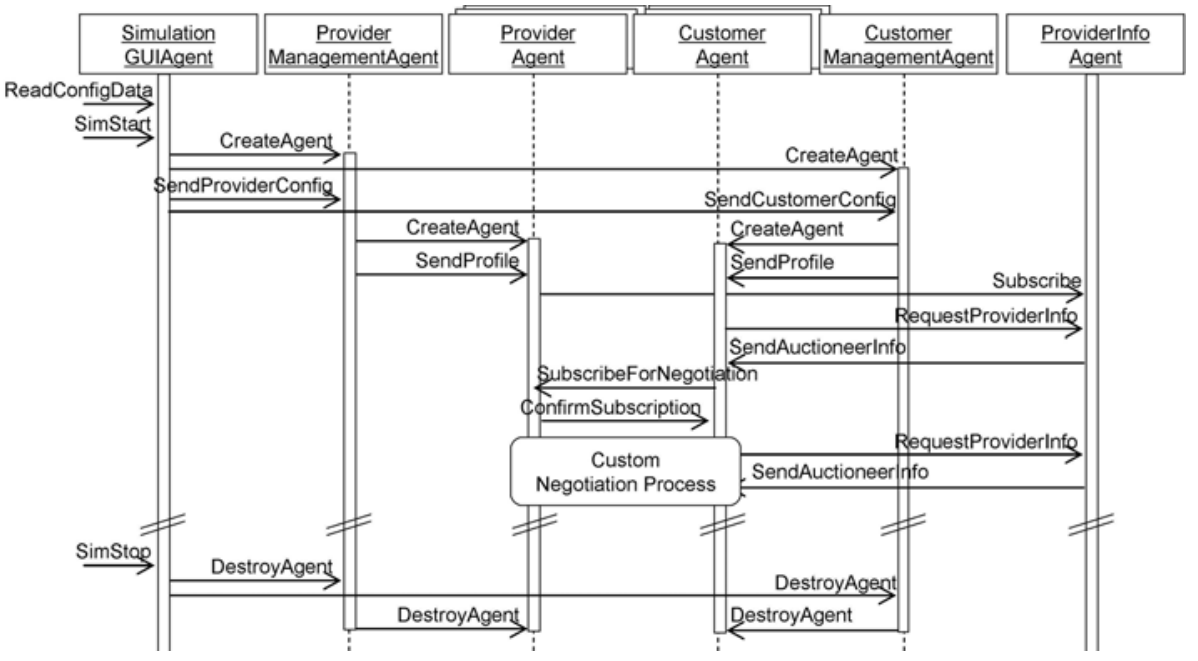

Fig. 2. Simpli ed sequence diagram of the simulation setup phase

Nash-Equilibrium the overall social welfare of the system is maximised. Social welfare is measured as the payoff from all agents including the providers' payoffs in the form of revenue obtained from the auction. We have developed alternative bidding strategies and compared the results with the optimal strategy in different network setups and user types.

\section{A. Experimental results}

In the following we brie y describe two basic experiments, using the PSP implementation, which have been run on the simulator. The reader is referred to [12] and [3] for an extended description of the auction mechanism and the bidding strategies implemented. These papers also elaborate on experimental results obtained by using the simulation environment.

In the rst experiment the bidding behaviour of a single agent can be observed. Figure 3 shows the requested share of resources for a bidder with elastic demand, who has access to two different providers. Both providers offer the same amount of resources but the load in both networks varies as other bidders join or leave the two networks. After the initial convergence phase the bidder is able to secure resources from both providers with the major share from provider 2. After new users join network two at 100 seconds the bidder shifts demand to provider 1. After each change in network ows, the user adjusts his bids to the individually optimal resource allocation.

The second experiment demonstrates how the simulation environment can be used to aggregate and process simulation results from single agents. We simulate a scenario with two different wireless networks and 10 bidders with different user types. All of them have access to both networks. Each network offers a different amount of network resource (which could be represented, e.g., by raw bandwidth or code slots).

Figure 4(b) shows the revenue obtained by each provider and the overall utility of all agents (including the utility of the provider agents) for each time step during the simulation. The aggregated utility shown in Figure 4(a) increases until the allocation reaches equilibrium at which time the welfare

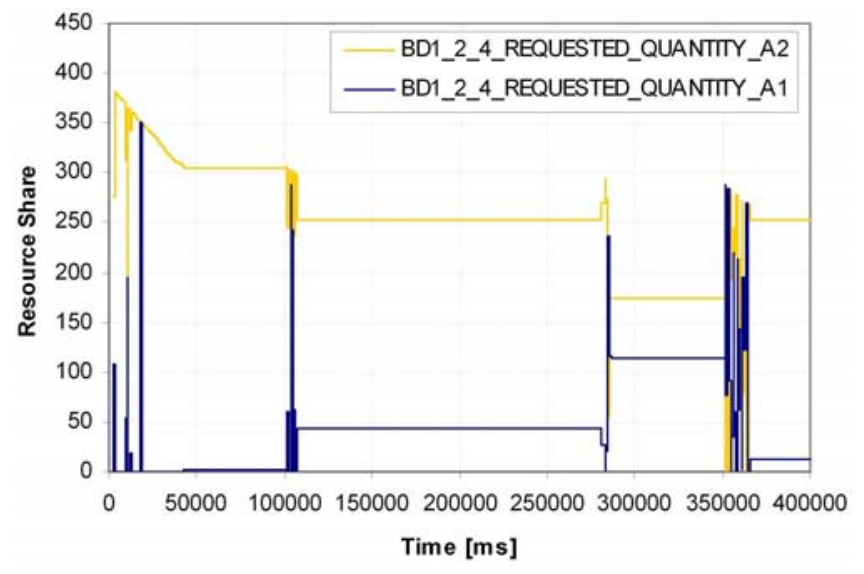

Fig. 3. The requested resource shares of a bidder with elastic demand with other bidders joining and leaving the network

measure is maximised. The revenue of both providers rst increases until it drops to a lower value on which the equilibrium is found. This behaviour can be explained with the customer agents submitting bids with higher prices but lower resource shares. Since in a second-price auction, the cost for each participant is based on the bids by other bidders, the revenue decreases since bidders start to reduce their requested shares. The extent of re-bidding is in uenced by the bid fee $\epsilon$, which is charged to a customer agent once it submits a new bid. With a bid fee of $\epsilon=0$ the revenue of a provider tends toward zero.

\section{B. Integration of PSP in the simulation environment}

Graphical output in the form shown here can be generated by the simulation environment at simulation runtime. Experiment data stored in the SQL database is converted to an Excel le and a graphic is generated automatically. Alternatively, data from past experiments can be accessed after the simulation to conduct more detailed analyses.

Two main classes had to be developed for implementing the speci c protocol of the PSP auction: a bidding engine class, which allows a customer agent to apply a speci c bidding 


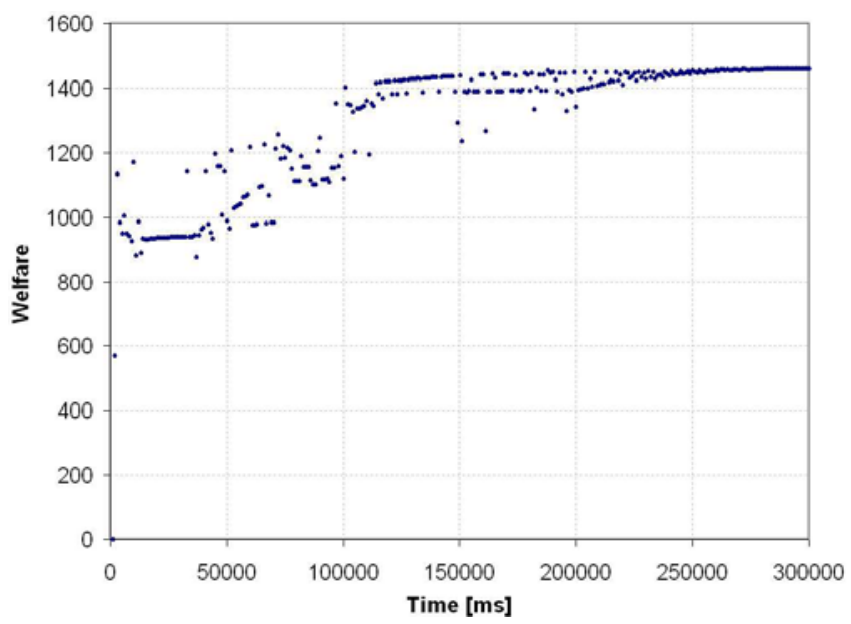

(a) Aggregated utility obtained by the resource allocation

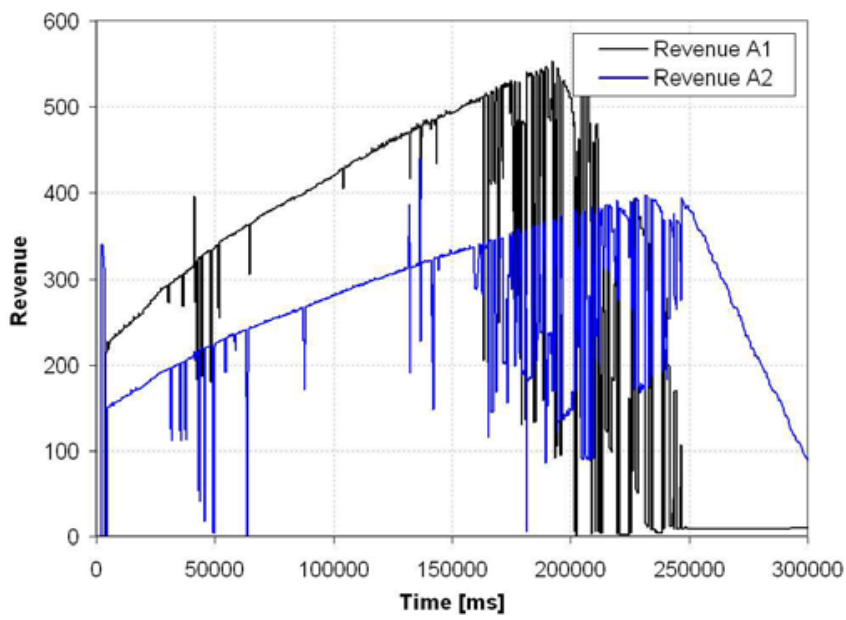

(b) Revenue generated by each provider

Fig. 4. Aggregated utility and provider revenue generated by the simulation platform with the described PSP auction mechanism (10 customer agents with random types, two provider agents, quantities $q_{1}=60, q_{2}=40$, bid fee $\epsilon=0.01$, smallest bidding unit $\alpha=0.1$ )

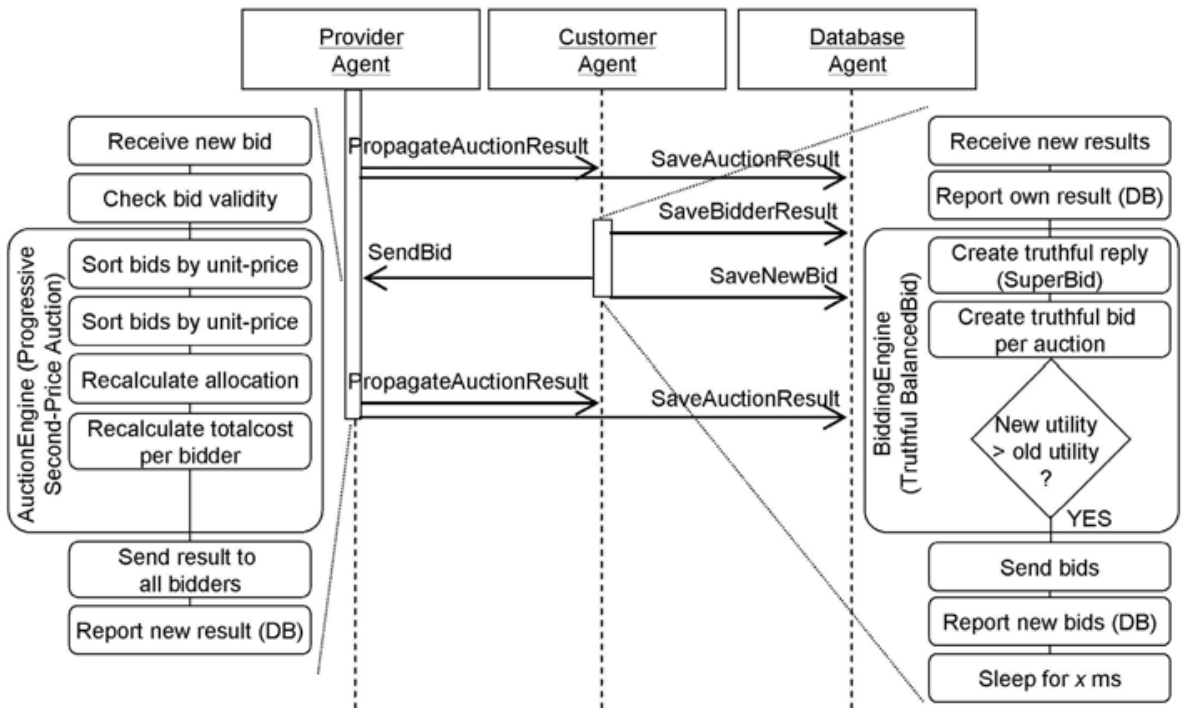

Fig. 5. The implementation of the second-price (PSP) auction protocol as a combined sequence/state diagram

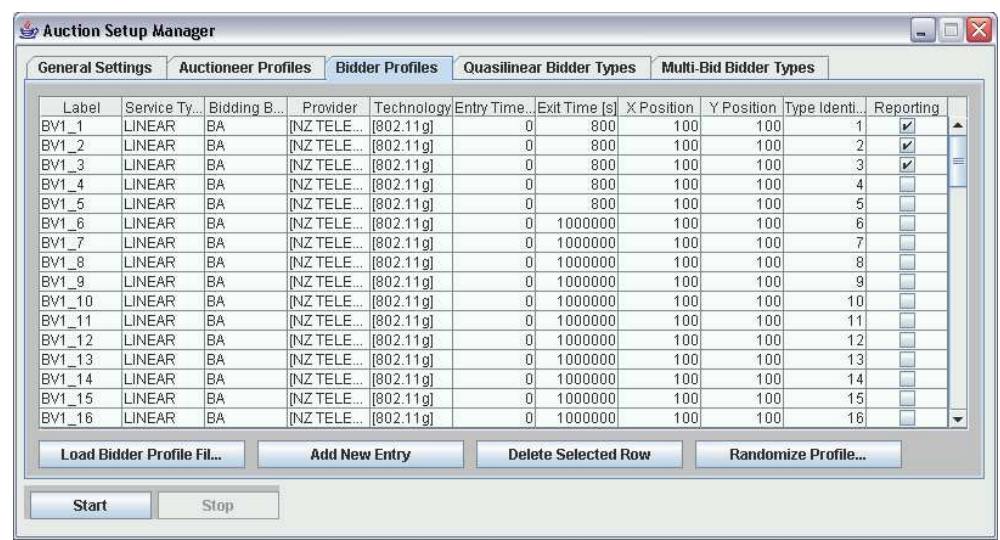

Fig. 6. The PSP auction GUI created for customising the experiments

strategy, and an auction engine class to run the PSP auction on the provider agent side.

The bidding engine class implements different bidding strategies for a customer agent. Depending on the con guration parameter of an agent, it uses such bidding strategies to calculate the bid for the next round of the auction. As 
soon as a customer agent receives an updated allocation from the provider agent, it forwards this information to the bidding engine, which provides it with a new bid to be submitted to one or more provider agents. For example, a possible bidding strategy may implement the objective to obtain maximum resources from only one provider. One way the strategy works is to analyse the competitive structure at each provider and to identify the auction with the highest utility for the agent.

The auction engine is used by all provider agents to calculate the resource allocation when at least one new bid has been received. The provider agent forwards the new bids to the auction engine, which recalculates the allocation. The implementation is based on the mechanism described in [11]. Figure 5 provides a graphical overview of the implementation of the PSP negotiation protocol in the simulation environment.

A graphical user interface (GUI) has been custom-built for this set of experiments to define agents representing customers, and agents playing the role of wireless access points (Figure 6). During the simulation run a second GUI displays the simulation status and allows direct access to the data collected during the simulation.

\section{CONCLUSIONS AND FUTURE WORK}

We have presented our simulation environment for dynamic price formation in next generation wireless networks at its first stage of development. The core architecture of the simulator has been implemented and was used for a first set of experiments with an auction-based allocation mechanism. All elements of the simulation environment have been defined as generic as possible to allow for a large class of allocation mechanisms to be used for pricing in similar scenarios. Open interfaces and a generic data collection method have been developed to allow for collecting data of different kinds from various entities in the system.

The next protocol to be implemented will be a multidimensional reverse auction mechanism. Users will be able to request network resources (e.g., in the form of service flows) with certain quality parameters such as guaranteed minimum bandwidth or maximum delay. Providers can send their offers (which can also be interpreted as bids) to the customer agent.
Each customer agent then holds an auction to determine the best offer from all providers. The main class to be developed for this set of experiments will be a resource planning engine on the provider side to maximise revenue, with offers sent out to potential customers. It is planned to mix users with subscription-based tariffs with users that will be able to dynamically negotiate for network resources. The objective of this research is to understand the potential of the dynamic pricing approach on the revenue achieved by providers. The final goal of the research is to develop a "Wireless Network Revenue Simulator" with which providers are able to evaluate innovative pricing plans in various competitive scenarios.

\section{REFERENCES}

[1] R. Berezdivin, R. Breinig, and R. Topp, "Next-generation wireless communications concepts and technologies," IEEE Communications Magazine, vol. 40, no. 3, pp. 108-16, 2002.

[2] I. Akyildiz and S. Mohanty, "A ubiquitous mobile communication architecture for next-generation heterogeneous wireless systems," IEEE Radio Communications, vol. 43, no. 6, pp. 29-36, 2005.

[3] M. R. F. Beltran, "Defining the optimal bidding function for divisible objects in multiple, simultaneous auctions." Unpublished working paper, October 2005. [Online]. Available: http://dream.com.auckland.ac.nz

[4] C. Courcoubetis and E. Weber, Pricing communication networks: economics, technology and modeling. Wiley-Interscience series in systems and optimization, 2003.

[5] 3rd Generation Partnership Project, "Technical Specification Group Core Network; Open Service Access (OSA); Application Programming Interface (API)," Tech. Rep. [Online]. Available: http://www.3gpp.org/

[6] Parlay Group, "Parlay API Specifications Version 5," Tech. Rep. [Online]. Available: http://www.parlay.org/specs/index.asp

[7] V. S. Frost, "Modeling and simulation in network management," Telecommunications Network Management into the 21st Century, IEEE Press, pp. 160-199, 1994.

[8] F. Bellifemine, G. Caire, T. Trucco, and G. Rimassa, "JADE Programmers Guide. Last Revision March 2005," Tech. Rep., 2005. [Online]. Available: http://jade.tilab.com/doc/programmersguide.pdf

[9] D. C. Parkes, "Iterative combinatorial auctions: Achieving economic and computational efficiency," Ph.D. dissertation, University of Pennsylvania, Chapter 2, 2001

[10] V. Krishna, Auction theory. Academic Press, 2002.

[11] N. Semret, "Market mechanisms for network resource sharing," Ph.D. dissertation, Columbia University, Center for Telecommunications Research, 1999.

[12] F. Beltran and M. Roggendorf, "A simulation model for the dynamic allocation of network resources in a competitive wireless scenario," in Proceedings of the Second International Workshop, MATA 2005, Montreal, Canada, October 17-19, 2005. Lecture Notes in Computer Science 3744 Springer, 2005. 